\title{
SIGNAL RESTORATION FOR A MASS TRANSPORT PROBLEM INVOLVING SHEAR DISPERSION
}

\author{
Paul R. Shorten and David J.N. Wall \\ Biomathematics Research Centre \\ Department of Mathematics \& Statistics \\ University of Canterbury, Private Bag 4800 \\ Christchurch, New Zealand
}

No. 163

March, 1998

Email address of D.J.N. Wall: D.Wall@math.canterbury.ac.nz

Email address of P.R. Shorten: P.Shorten@math.canterbury.ac.nz

1995 Physics and Astronomy Classification Scheme: PACS: 02.30Jr, 02.30Rz, 03.40Gc, 87.45 


\title{
SIGNAL RESTORATION FOR A MASS TRANSPORT PROBLEM INVOLVING SHEAR DISPERSION
}

\author{
PAUL R. SHORTEN AND DAVID J. N. WALL
}

\begin{abstract}
An inverse problem associated with mass transport down a tube, when the flowing medium has a two-dimensional velocity profile, is examined. The inverse problem of estimation of a temporally varying concentration at one end of a long tube, from the measurement of the cross-sectional average concentration at the opposite end, is solved. It is shown that this inverse problem, which is associated with shear dispersion, is an ill-posed deconvolution problem. Mollification is used to produce a well-conditioned problem.
\end{abstract}

\section{INTRODUCTION}

We consider an inverse problem associated with mass transport of a material concentration down a tube, when the flowing medium has a two-dimensional velocity profile. The fluid flow transports a concentration of suspended material down the tube, and its concentration is measured downstream. From this measured concentration the temporal variation of the concentration at an upstream location is to be estimated.

In viscous flow problems, involving a solute concentration in a tube, the variation of the velocity over the cross-section of the tube is an important factor in the dispersion of the solute; it is called shear dispersion. The dispersion of a pulse of concentration flowing down the tube is in general due to the combined action of shear dispersion, parallel to the axis of the tube, and molecular diffusion, predominantly in the radial direction. When the molecular diffusion coefficient within the flow is very small, then the fluid shear dispersion is the dominant dispersive effect. It is this regime that is examined in this paper.

The first paper providing an approximate analysis of the direct problem related to these effects is due to Taylor [1], and a considerable literature has built up about this direct problem (for recent application of modern theory to this direct problem see [2]). Of interest for inverse problem practitioners is Taylor's paper [3], in which he estimates the molecular diffusion coefficient from measured concentration profiles, after dispersion. This inverse problem solution is one of the earliest inverse problem investigations in this area.

The model problem, considered in this paper, has practical application in an experimental apparatus used in endocrinology experiments, where it is required to estimate temporal changes in concentration, upstream, from temporal concentrations changes measured at the end of a long tube. Information transfer in a number of endocrine systems occurs through rapid modulation of hormone levels in concentration pulses. Experimental methods of investigating how the dynamics of signal transmission in endocrine systems relates to the mechanisms involved is often carried out in vitro by use of a perifusion apparatus $[4,5]$. In this apparatus measurement of averaged hormone concentration is made downstream, along a tube (up to a few metres in length) from the pituitary cells which are attached to micro-beads. The flow

Date: 27 March 1998.

1995 Physics and Astronomy Classification Scheme. PACS: 02.30Jr, 02.30Rz, 03.40Gc, 87.45 . 
rate of the carrying medium, a saline preparation, in the tube is low and the internal diameter of the tube is small. Longitudinal molecular diffusion effects of the hormone concentration suspended in the saline solution are small with respect to the mixing due to two-dimensional velocity distribution, for this problem. In fact for the molecular particles and temperatures used in the in vitro experiment described earlier longitudinal molecular diffusion is several orders of magnitude less dominant. A model of the perifusion apparatus as a one-dimensional convective-diffusive flow problem has been provided by [6]. This model neglects radial dispersion caused by both shear dispersion and cross-diffusion effects. The analysis of the inverse problem associated with this simplified one-dimensional model was done in [7].

Inverse mass transport problems involving one-dimension flow, but without diffusion, have also been analysed in [8], where inverse source problems associated with linear transport equations were solved. Various inverse problems associated with nonlinear transport equations have also been described in [9].

This paper concentrates on the inverse problem when the dispersion of the concentration of contaminant is due solely to the velocity profile. In $\S 2.1$ the equations modelling the flow of hormone concentration down the tube are given, and the mathematical operator mapping the temporally varying concentration to the mixed, and experimentally measured, temporally varying concentration is examined. In $\S 2.2$ it is shown that the inverse of this operator is unbounded on the Hilbert space of square integrable functions, $L^{2}$, and it is shown that the deconvolution problem, described by this operator, is an ill-posed problem. In $\S 3$ the mollification method is used to regularise the inverse problem, and the stability of the regularised solution is analysed. Numerical results are presented in $\S 4$, where it is shown that the regularised deconvolution problem can be effectively solved, even in the presence of a moderate amount of measurement noise.

\section{Problem Description}

The two-dimensional laminar flow model is first analysed, and then the inverse problem is described.

2.1. Basic Equations describing the Mass Transport. The velocity profile of a viscous, incompressible fluid flowing through a rigid tube of circular cross-section with a no-slip boundary condition, is described by the well known Poiseuille distribution [10], (pages 57 et. seq.)

$$
v(r)=v_{m}\left(1-\left(\frac{r}{R}\right)^{2}\right)
$$

where $r$ is the circular polar radial coordinate associated with a cylindrical coordinate system oriented so that the $z$-axis is aligned with the axis of the tube, and the wall of the tube is located at $r=R$. The maximum velocity of the parabolic velocity profile, $v_{m}$, depends upon the pressure differential applied down the tube. The fluid flowing in the tube is assumed to be Newtonian, and have a suitably low Reynolds number ${ }^{1}$ so that the fluid flow is laminar.

The mass transport, of a volume concentration of a hormone $c(z, r, t)$ down the tube, is described by the transport equation (one-way wave equation),

$$
\frac{\partial c}{\partial t}+v(r) \frac{\partial c}{\partial z}=0, \quad z \in[0, \infty), \quad r \in[0, R], \quad t>0,
$$

\footnotetext{
${ }^{1}$ For a saline solution typically used in perifusion experiments the Reynolds number is of order 1.
} 
where the advection velocity distribution is prescribed by (2.1). This equation is a Cauchy initial value problem with the specification of the boundary value $c(0, r, t)$, which is assumed to be independent of $r$, that is, it is assumed that the concentration is uniformly distributed across the cross-section of the tube at $z=0$. Because of this assumed radial independence we write $c(0, r, t) \equiv c_{0}(t)$. This assumption is essential for the development of the method presented in this paper.

When the concentration level in the tube is initially zero, $\left.c(z, r, t)\right|_{t=0}=0$, then elementary integration of the equation (2.2) shows that

$$
c(z, r, t)=c_{0}\left(t-\frac{z}{v(r)}\right) \mathrm{H}\left(t-\frac{z}{v(r)}\right), \quad z \in[0, \infty), \quad r \in[0, R], \quad t>0,
$$

where $\mathrm{H}$ denotes the Heaviside step function. If it is possible to measure this concentration, $c\left(\ell, r_{1}, t\right)$, at a fixed station, say $z=\ell$ and $0 \leq r_{1}<R$, downstream of the injection point, then estimation of the injection temporal profile, $c_{0}(t)$, is a straightforward well-posed problem; see [8] for discussion of such hyperbolic inverse problems. However, generally the concentration, at the station $z=\ell$, must be assayed over the whole tube. The simplest inverse problem will then require the measurement of an average concentration, $Q(t)$, across the cross-section of the tube. Simple analysis of the geometry and (2.3) then shows that

$$
Q(t)=\frac{2}{R^{2}} \int_{0}^{R} c_{0}\left(t-\frac{\ell}{v(r)}\right) r d r
$$

This is the case studied in $\S 2$ of [1]. It is readily appreciated that the operator defined in this equation is a smoothing operator. The exact nature of which is easily seen on application of the transformation $s=t-\frac{\ell}{v(r)}$ to the integral in (2.4), so yielding

$$
Q(t)=\frac{\ell}{v_{m}} \int_{-\infty}^{t-\frac{\ell}{v_{m}}} \frac{c_{0}(s)}{(t-s)^{2}} d s .
$$

This equation can be further reduced to the more standard form

$$
Q(t+a)=\int_{-\infty}^{t} k(t-s) c_{0}(s) d s
$$

where the kernel has the functional form

$$
k(t)=\frac{a}{(t+a)^{2}}, \quad \text { with } \quad a=\frac{\ell}{v_{m}}>0 .
$$

The parameter $a$ represents the time that the fluid travelling with the maximum speed $v_{m}$ takes to reach the station at $z=\ell$.

2.2. Inverse Problem. It is now straightforward to examine the stability of the inverse problem of estimation of the function $c_{0}$, from knowledge of $Q$. It should be first observed that (2.5) is a Volterra equation of the first kind, with a regular kernel, which is a well known ill-posed problem [11], (pages 161 et. seq.). To understand the degree of ill-posedness it is convenient to perform Fourier analysis of (2.5). To this end, extend the data function $Q(t)$, and the kernel function $k(t)$, by zero for $t<0$, and consider their Fourier transforms. The Fourier transform of $t \quad$.el is pivotal in our argument; it is

$$
\widehat{k}(\xi)=\frac{a}{\sqrt{2 \pi}} \int_{-\infty}^{\infty} \frac{\mathrm{H}(t) \exp (-i \xi t)}{(t+a)^{2}} d t
$$


and on integrating by parts, this transform is converted to

$$
\widehat{k}(\xi)=\frac{a}{\sqrt{2 \pi}}\left[\frac{1}{i \xi a^{2}}-\frac{2}{i \xi} \int_{-\infty}^{\infty} \frac{\mathrm{H}(t) \exp (-i \xi t)}{(t+a)^{3}} d t\right] .
$$

By continuing this procedure, an asymptotic series in reciprocal powers of $\xi$ is obtained, as

$$
\widehat{k}(\xi)=\frac{1}{\sqrt{2 \pi}}\left(\frac{1}{(i \xi a)}-\frac{2}{(i \xi a)^{2}}\right)+\mathcal{O}\left((i \xi a)^{-3}\right) .
$$

It should be noted that the dominant term in the asymptotic expansion of $\widehat{k}(\xi)$, as $|\xi| \rightarrow \infty$, is therefore $1 /(i a \sqrt{2 \pi} \xi)$. Therefore from (2.5), using the convolution theorem, it follows that $Q$ is not just a function in $L^{2}(\mathbb{R})$, but its high frequency behaviour is such that $\|\widehat{Q}\|_{2}$ decreases faster than $\left|\xi^{-1}\right|$, as $|\xi| \rightarrow \infty$, because by Parseval's theorem $c_{0} \in L^{2} \Longleftrightarrow \widehat{c_{0}} \in L^{2}$. It is readily observed that for a general noise function, $n(t) \in L^{2}(\mathbb{R})$, assumed to be additive to $Q(t)$, there is no reason to believe that the high-frequency components of $\widehat{n}(\xi)$ will be subject to such rapidly decreasing behaviour, and it therefore follows that there is no guarantee that the product $\xi \widehat{n}(\xi)$ will be in $L^{2}(\mathbb{R})$. This illustrates that the deconvolution problem associated with the solution of the inverse signal reconstruction is ill-posed. It also follows from the above asymptotic analysis that the inverse problem is equivalent to differentiation, and it is therefore necessary to restore continuity with respect to the data in order to successfully solve the problem.

An alternative approach to directly solving (2.5) is to differentiate this equation, so leading to

$$
Q^{\prime}(t+a)=\frac{1}{a} c_{0}(t)+\int_{-\infty}^{t} \tilde{k}(t-s) c_{0}(s) d s,
$$

where the kernel of this Volterra equation of the second kind is, $\tilde{k}(t-s) \equiv k^{\prime}(t-s)=-2 a /(t-$ $s+a)^{3}$, and the prime denotes differentiation with respect to the function argument. This equation re-enforces the idea that the inversion of the integral equation (2.5) has numerical conditioning equivalent to differentiation. If we denote the function space of $n$-th order continuously differentiable functions by $C^{n}$, and note that as the kernel $\widetilde{k}$ is in $C[0, T]$, the integral operator in equation (2.6) is of compact Volterra type, mapping $C[0, T]$ into itself, and similarly for the operator in (2.5). Given that $Q$, on the left-hand-side of equation (2.6), is assumed to be in $C^{1}$, then there exists a unique solution $c_{0} \in C[0, T]$ of the integral equation (2.6) [11], (pages 30 et. seq.). Equation (2.6) is the basis of our deconvolution method to solve the inverse problem of estimation of $c_{0}$ from the measurement of $Q$.

Equation (2.6) defines the operator mapping the concentration signal $c_{0}(t)$ to the measured average flow $Q(t)$, that is $\mathbb{T}: c_{0}(t) \mapsto Q(t)$. It is the inverse of this map that defines the mathematical properties of the inverse problem, that is $\mathbb{T}^{-1} Q \mapsto c_{0}$. As the differentiation operator is unbounded on the function space $C$, and as further explained in $\S 3$ that is the space we must work, it follows that this operator is unbounded. Similar reasoning shows that this operator is unbounded on $L^{2}$. The regularisation of the inverse operator $\mathbb{T}^{-1}$ is the subject of the next section.

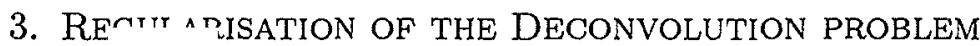

The inverse problem of source concentration reconstruction presented in $\S 2.2$ is considered here. This inverse problem is always ill-posed for realistic measurement data. This is because data that is measured, can generally only be placed in the function space $L^{2}$, or at most 
$C$, and in these function spaces differentiation operators are unbounded. As shown in $\S 2.2$, the inverse operator mapping the measurement to the solution involves differentiation. It is therefore central to our treatment to show that numerical differentiation can be made a wellposed problem. That this can be done is well known, and there are a number of regularisation techniques available. We shall choose the method of mollification in this paper, and we follow the treatment of Murio [12].

The problem is to get a regularised approximation to the derivative of $f$ when given a modified function $f_{m}$; where due to measurement difficulties the true function $f$, has been corrupted by a noise function $n$, so that

$$
f_{m}(x)=f(x)+n(x), \quad x \in I .
$$

The functions $f$, and $n$, are defined on the interval $I=[0, T]$, for a given value of $T>0$.

Consider the mollification, or Gaussian function

$$
\rho_{\delta}=\frac{1}{\delta \sqrt{\pi}} e^{-x^{2} / \delta^{2}}, \quad x \in \mathbb{R}
$$

The important properties of this function are:

1. $\rho_{\delta} \in C^{\infty}$ and is nearly compact, as it is almost zero outside $|x|<3 \delta$.

2. $\int_{\mathbb{R}} \rho_{\delta}(x) d x=1$, whereas $\int_{-3 \delta}^{3 \delta} \rho_{\delta}(x) d x \cong 0.997$.

To proceed, extend the data $f_{m}$ to the interval $I_{\delta}=[-3 \delta, 3 \delta+T]$ by

$$
\begin{aligned}
& f_{m}(x)=f_{m}(0) \exp \left[x^{2} /\left[x^{2}-(3 \delta)^{2}\right]\right], \quad-3 \delta \leq x<0, \\
& f_{m}(x)=f_{m}(T) \exp \left[(x-T)^{2} /\left[(x-T)^{2}-(3 \delta)^{2}\right]\right], \quad T<x \leq T+3 \delta,
\end{aligned}
$$

and then define the mollifier of $f$ by

$$
\begin{aligned}
J_{\delta} f(x) & =\left(\rho_{\delta} * f\right)(x)=\int_{-\infty}^{\infty} \rho_{\delta}(x-s) f(s) d s \\
& \approx \int_{x-3 \delta}^{x+3 \delta} \rho_{\delta}(x-s) f(s) d s
\end{aligned}
$$

where $\delta>0$ is the radius of mollification. We define the norm $\|f(t)\|_{\infty}=\sup _{t \in I}|f(t)|$, then the following results are central to our stability proof.

Lemma: Murio's Consistency. If $\left\|f^{\prime \prime}\right\|_{\infty} \leq M_{2}$ then

$$
\left\|\left(J_{\delta} f\right)^{\prime}-f^{\prime}\right\|_{\infty} \leq 3 \delta M_{2} \text {. }
$$

This consistency result shows that as $\delta \rightarrow 0$, then $\left(J_{\delta} f\right)^{\prime} \rightarrow f^{\prime}$.

Lemma: Murio's Stability. With $f_{m} \in C\left(I_{\delta}\right)$

$$
\left\|\left(J_{\delta} f\right)^{\prime}-\left(J_{\delta} f_{m}\right)^{\prime}\right\|_{\infty} \leq \frac{2}{\delta \sqrt{\pi}}\left\|f_{m}-f\right\|_{\infty}
$$

We see the mollification method provides the differentiation operator with a Lipschitz continuity result, when the data $f_{m} \in C$, provided $\delta>0$ is fixed. Furthermore as $\left\|f_{m}-f\right\| \rightarrow$ $0, \delta$ can be reduced, a consistency error is then decreased, provided $f \in C^{2}$.

We now define the regularised inverse source problem. First mollify the measurement data that is specified in $\S 2.2$, by forming $J_{\delta} Q(t)$, and then solve the inverse source problem with this regularised measurement data, through (2.6). 


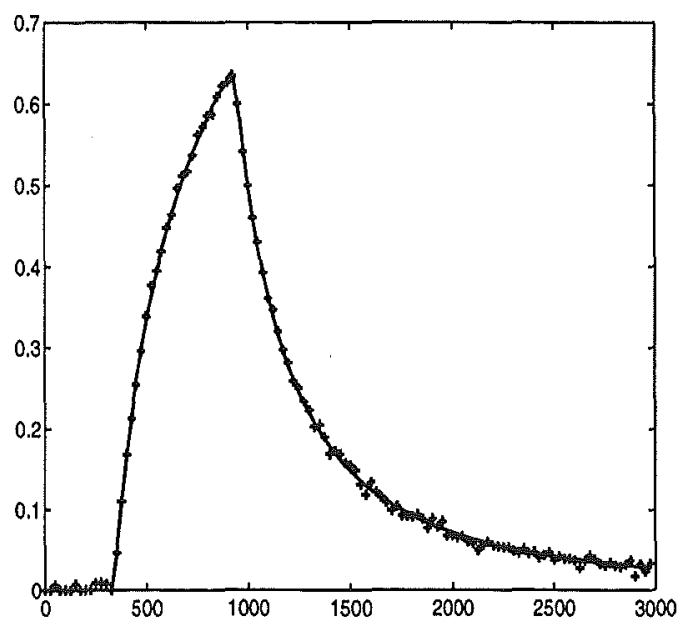

(a) The simulated measured time signal, $Q$, with no noise present _ and with $2.5 \%$ noise present, $Q_{m}=Q+n$, as indicated by ++ .

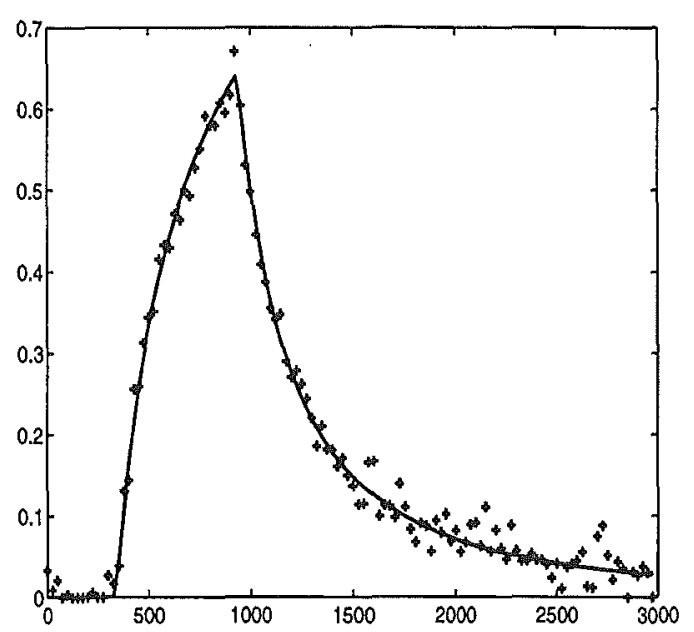

(c) The simulated measured time signal, $Q$, with no noise present $\longrightarrow$ and with $5 \%$ noise present, $Q_{m}=Q+n$, as indicated by ++ .

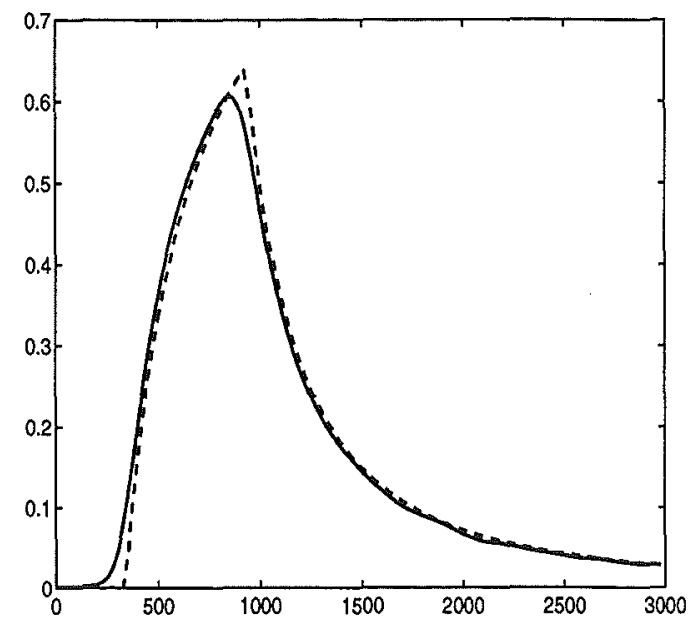

(b) The simulated measured time signal, $Q$, with no noise present - - - and the mollified time signal, $J\left(Q_{m}\right)$ — with $2.5 \%$ mollification when $2.5 \%$ noise is present on $Q_{m}$.

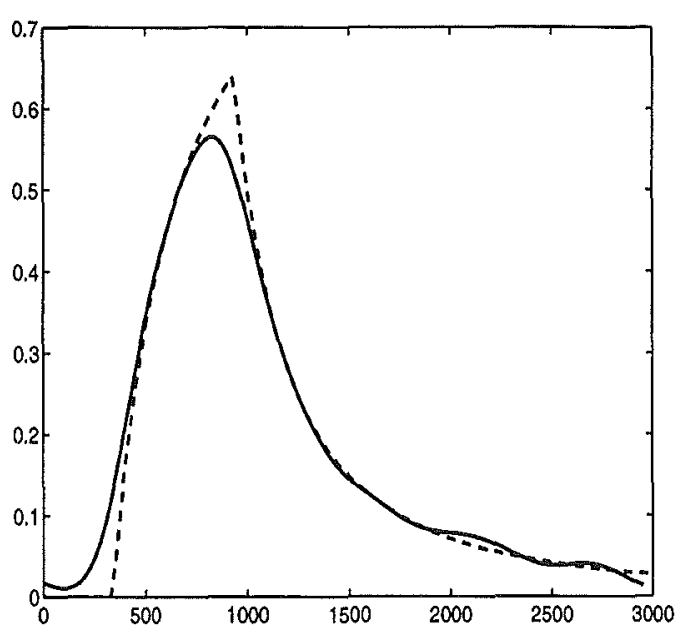

(d) The simulated measured time signal, $Q$, with no noise present - - - , and the mollified time signal, $J\left(Q_{m}\right)$ with $5 \%$ mollification when $5 \%$ noise is present on $Q_{m}$.

FIGURE 1. The measured temporal signal $Q(t)$, the noisy signal $Q_{m}(t)$, and the mollified $\operatorname{si}^{m n n}{ }^{\top}\left(Q_{m}\right)(t)$.

Theorem 3.1. The signal reconstruction problem, as stated in $\S 2.2$, with mollified measurement data $J_{\delta} Q$ has a well posed solution, provided that $Q_{m} \in C[0, T]$. 
Proof. We denote the measured function value that has not been corrupted by noise, by $Q$, and the modified value due to the noise by $Q_{m}$; the actual measurement.

We first must bound the appropriate Volterra operators, before using the stability results from the beginning of this section for the differentiation operator. Consider firstly the equation

$$
(\lambda \mathbb{I}-\mathbb{K}) v=f
$$

where $\mathbb{K}$ is a linear compact Volterra integral operator in the function space of continuous functions defined on an appropriate interval, and secondly the perturbed mapping equation

$$
(\lambda \mathbb{I}-\mathbb{K}) w=f+\Delta f .
$$

In this equation $f$ and $\Delta f$ are some appropriate continuous functions, and it is assumed that the operator $\mathbb{K}$ has a continuous kernel $\widetilde{k}$, as given in (2.6). Then standard theory (see for example [11], (pages 45 et. seq.)), shows that if there exists two constants $K$ and $\Delta$, such that $|\widetilde{k}|<K,|\Delta f|<\Delta$, and if $\lambda$ is a constant, then

$$
|v(t)-w(t)| \leq \frac{\Delta}{\lambda} \exp (K t / \lambda)
$$

where for (2.6), $K=-2 / a^{2}$, and $\lambda=1 / a$. It follows, with $\mathbb{T}^{-1}$ defined as $\mathbb{T}^{-1} f=(\lambda \mathbb{I}-\mathbb{K})^{-1} f$, together with mollification of the measurement function, $Q_{m}$, and by Murio's stability lemma, we have the Lipschitz continuity result

$$
\left\|\mathbb{T}^{-1}\left(J_{\delta} Q\right)^{\prime}-\mathbb{T}^{-1}\left(J_{\delta} Q_{m}\right)^{\prime}\right\|_{\infty} \leq \frac{2 \exp (K T / \lambda)}{\lambda \delta \sqrt{\pi}}\left\|Q_{m}-Q\right\|_{\infty}
$$

Consistency of the mollified problem solution to the exact $c_{0}$ can be shown from Murio's consistency lemma, provided that $Q(t) \in C^{2}[0, T]$. Examination of (2.5) shows, with the assumption that $c_{0} \in C^{1}[0, T]$ and because of the smooth kernel, $k$, that $Q \in C^{2}[0, T]$.

\section{Numerical Method AND Results}

In the results presented in this section the various parameters are taken from the perifusion experimental setup associated with $\mathrm{ACTH}{ }^{2}$ measurements $[13,14]$, and they are: $v_{m}=$ $6 \times 10^{-3} \mathrm{~ms}^{-1}, \ell=2 \mathrm{~m}, R=0.5 \times 10^{-3} \mathrm{~m}$, so that $a=1 / 3 \times 10^{3} \mathrm{~s}$. The measured time signal $Q(t)$ for $0 \leq t \leq T$, with $T=3000 \mathrm{~s}$, is assumed to be the known function in the inverse problem. An important property of the problem is the finite speed of mass transport, so that $Q^{\prime}(t)=0$, when $t<a$. It is therefore assumed that $Q(t)=0$ for $t<a$, implying that $c_{0}(t)=0$ for $t<0$.

We now describe the numerical algorithm used to solve the inverse problem. Define the natural numbers $i, j, M, N \in \mathbb{N}$, and then a mesh $\left\{t_{i}\right\}_{i=0}^{N}$, with uniform mesh interval $h=$ $T / N$, and $t_{0}=0, t_{i}=t_{i-1}+h, \quad 1 \leq i \leq N$, is established on the time axis. The signal function $c_{0}(t)$ is now approximated by a B-spline of degree $n$, denoted by $\mathcal{S}_{n}$, such that

$$
c_{0}(t)=\sum_{i=0}^{M} \alpha_{i} b_{i}(t)
$$

\footnotetext{
${ }^{2}$ Adrenocorticotrophic hormone (ACTH) is the major hormone released from the corticotrophin cells in the pituitary gland in response to stimulation, and is often the hormone concentration measured in perifusion experiments.
} 


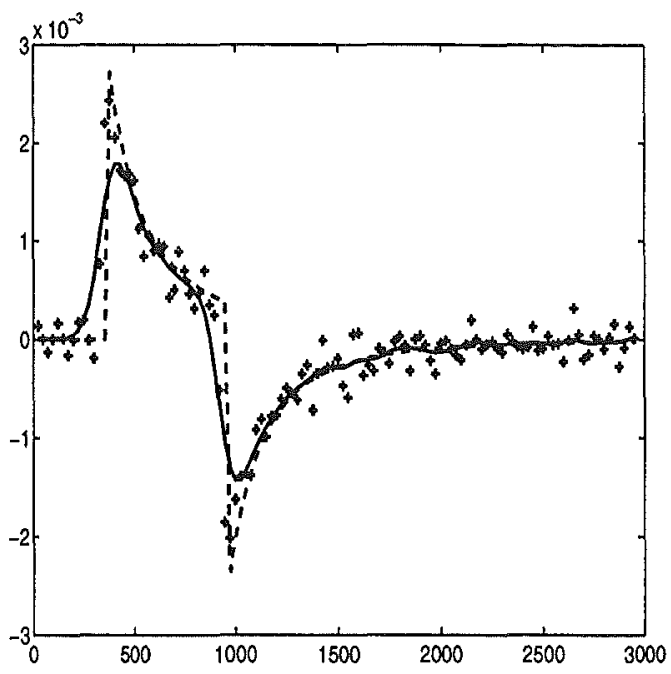

(a) The derivative of the measured time signal, $Q^{\prime}(t)$, with no noise present - - , the mollified derivative $\left(J\left(Q_{m}\right)\right)^{\prime}(t)$ with $2.5 \%$ mollification , and the direct derivative $Q_{m}^{\prime}(t)$, indicated by ++ . The noise level in $Q_{m}$ is $2.5 \%$.

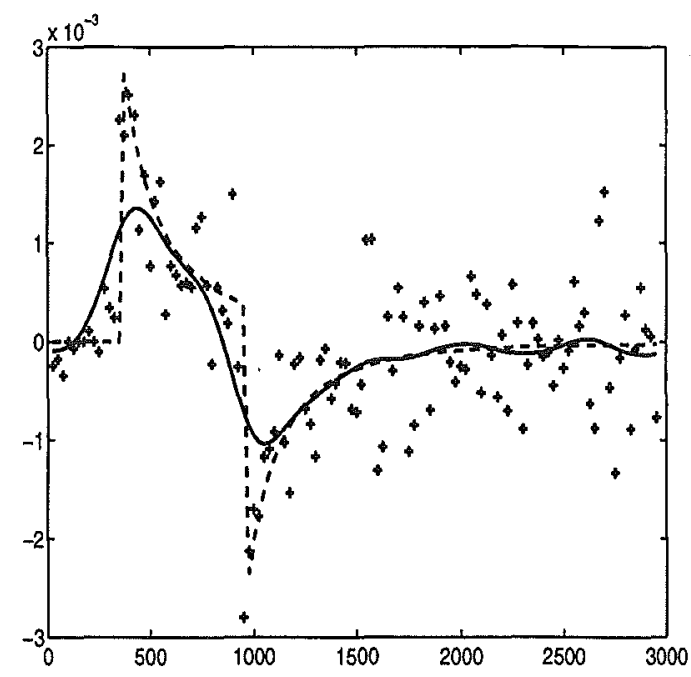

(b) The derivative of the measured time signal, $Q^{\prime}(t)$, with no noise present - - the mollified derivative $\left(J\left(Q_{m}\right)\right)^{\prime}(t)$ with $5 \%$ mollification $\longrightarrow$, and the direct derivative $Q_{m}^{\prime}(t)$, indicated by ++ . The noise level in $Q_{m}$ is $5 \%$.

Figure 2. Estimation of the derivative $Q^{\prime}(t)$ for noise levels of $2.5 \%$ and $5 \%$.

where $M+1$ is the cardinality of the B-spline basis, $\left\{b_{i}(t)\right\}_{i=0}^{M} \in \mathcal{S}_{n}[0, T]$. Collocation of the equation (2.6) then provides the finite dimensional equation

$$
\frac{\alpha_{j}}{a}+\sum_{i=0}^{M} \alpha_{i} \int_{-\infty}^{t_{j}} \tilde{k}\left(t_{j}-s\right) b_{i}(s) d s=Q^{\prime}\left(t_{j}+a\right), \quad j \in[0, N]
$$

The properties of the B-splines and their support implies that the matrix defined by this equation will be lower triangular. For a concrete implementation of the algorithm, the integral is approximated by the trapezoidal rule, and $n$ is chosen to be one; so that the B-spline basis functions are the piecewise linear, or hat, functions.

Now denote $Q^{\prime}\left(t_{i}+a\right), \widetilde{k}\left(t_{i}\right)$ and $(J(Q))^{\prime}\left(t_{i}\right)$ by $Q_{i}^{\prime}, \widetilde{k}_{i}$, and $(J(Q))_{i}^{\prime}$ respectively. Note for notational simplicity the subscript $\delta$ has been suppressed from the mollification operator $J$. Then (4.2) can be solved for the unknown coefficients $\left\{\alpha_{i}\right\}_{i=0}^{N}$, so yielding

$$
\begin{aligned}
& \alpha_{0}=a Q_{0}^{\prime}, \\
& \alpha \cdot-\left({Q_{j}^{\prime}}^{\prime}-h \sum_{i=1}^{j-1} \widetilde{k}_{i} \alpha_{i}\right) /\left(a+\frac{h}{2} \widetilde{k}_{0}\right), \quad 1 \leq j \leq N,
\end{aligned}
$$

where the prime on the summation sign is to signify that the last value in the summation is to be halved. 


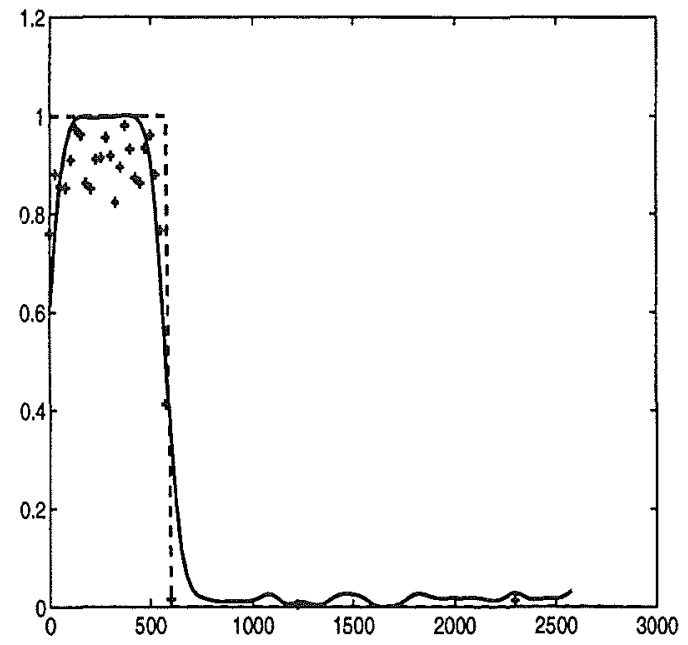

(a) The reconstructed time signal, co $(t)$. The mollified reconstructed $c_{0} \longrightarrow$, with $2.5 \%$ mollification, the directly reconstructed co ++ , and the exact $c_{0}---$. Both the former two reconstructions are from $Q_{m}$ with $2.5 \%$ noise present.

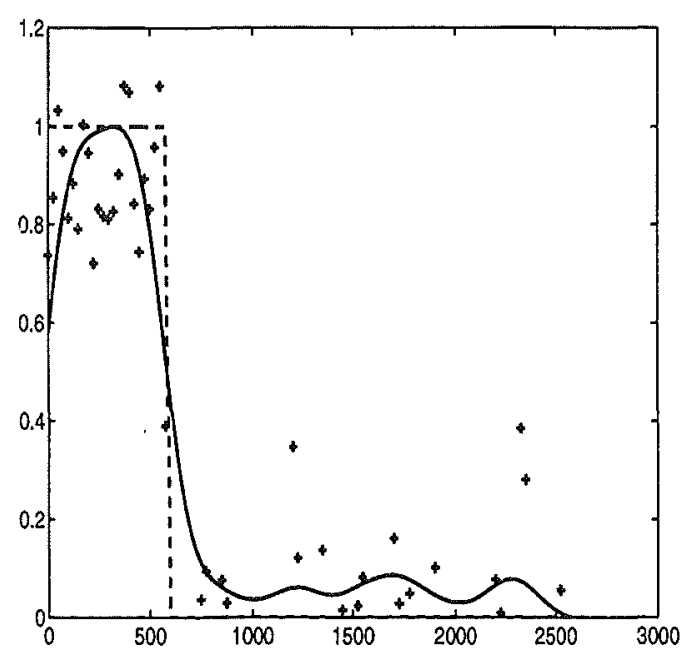

(b) The reconstructed time signal, $c_{0}(t)$. The mollified reconstructed $c_{0} \longrightarrow$, with $5 \%$ mollification, the directly reconstructed $c_{0}++$, and the exact $c_{0}---$. Both the former two reconstructions are from $Q_{m}$ with $5 \%$ noise present.

FIGURE 3. Reconstruction of the temporal boundary concentration $c_{0}$.

All that remains to ensure that the algorithm is well-posed, is to evaluate the coefficients $\left\{Q_{i}^{\prime}\right\}_{i=0}^{N}$ as the mollified derivative values, $\left\{\left(J_{\delta} Q\right)_{i}^{\prime}\right\}_{i=0}^{N}$, as described in $\S 3$, but with the standard numerical central differences formula used to estimate the numerical derivative.

4.1. Numerical Results. The measurement data utilised in this section was simulated through the use of equation (2.5). The calculated data $Q$ was corrupted with white noise having a normal distribution and zero mean, so that the averaged concentration, $Q_{m}=Q+n$ was used in the measured simulation. The quoted value of the noise level, in our results, is a relative measure of the standard deviation of the noise to $\|Q\|_{2}$, where $\|\cdot\|_{2}$ denotes the $\ell_{2}$ sequence space norm. In a practical experiment it is not possible to measure a negative concentration, so when $Q_{m}$ was found to be negative in the simulation, it was replaced by a zero value.

In Figure 1 the computer simulation of averaged concentrations at $z=\ell$ is illustrated for various noise levels. The boundary value, of the concentration at $z=0$, was chosen as representative of the initial hormone input into the tube, namely the pulse function

$$
c_{0}(t)=\mathrm{H}(t)-\mathrm{H}(t-L), \quad 0 \leq t \leq T,
$$

where $L=600 s$, an ' ' is the boundary value used throughout this paper. This figure shows how the mollification of the noisy signal provides a acceptable smoothing on the noisy data, and provides a good approximation to the main features of the clean signal. The time signal $Q(t)$ can be found analytically from (2.5), when the concentration at $z=0$ is given 


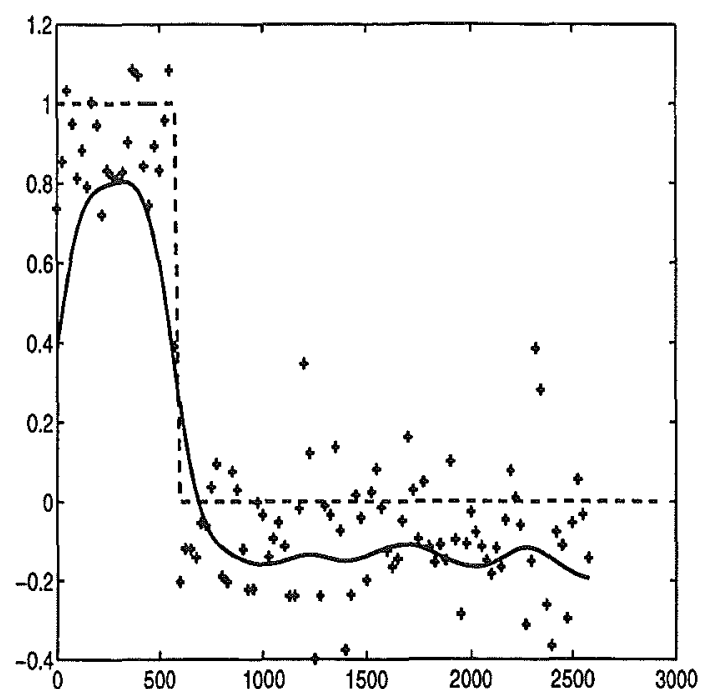

(a) The reconstructed time signal, $c_{0}(t)$. The mollified reconstructed $c_{0} \ldots$, with $5 \%$ mollification but without the positivity adjustment, the directly reconstructed $c_{0} t+$, and the exact $c_{0}---$. Both the former two reconstructions are from $Q_{m}$ with $5 \%$ noise present.

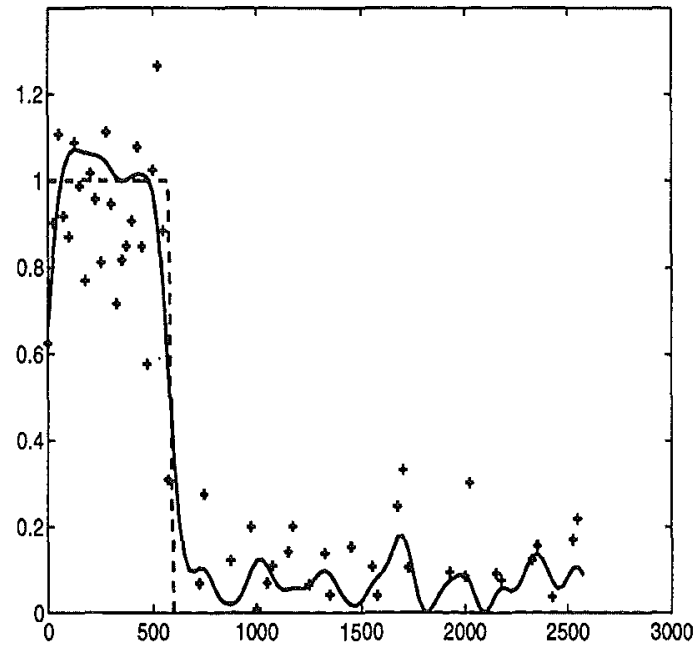

(b) The reconstructed time signal, $c_{0}(t)$. The mollified reconstructed $c_{0} \ldots$, with $2.5 \%$ mollification, the directly reconstructed $c_{0}++$, and the exact $c_{0}---$. Both the former two reconstructions are from $Q_{m}$ with $5 \%$ noise present.

FIGURE 4. Reconstruction of the temporal boundary concentration $c_{0}$.

by (4.4). In all simulated results presented in this paper $T=3000 \mathrm{~s}$ and $N=120$, so that $h=25$.

The amount of mollification utilised in our numerical reconstructions is stated as a relative percentage of $\delta / T$, with $T$ denoting the maximum time for which the data is collected. Another way of quoting this is to express the $\delta$ size in seconds. The value of the mollification parameter $\delta$, was generally chosen to be approximately the same relative percentage of $T$ as the relative percentage noise level. More sophisticated methods of optimising $\delta$ can be found in [12]. Although our experience indicates the choice of $\delta$ is not too critical provided $\delta>h$. Relative percentage values of mollification of $2.5 \%$ and $5 \%$, correspond to $\delta$ values of 75 and 150 , respectively, in this paper.

Figure 2 shows the directly estimated, the mollified, and accurate derivatives of the function $Q^{\prime}(t)$; these are used in equation (2.6) of our algorithm. It is seen from these figures, that for low noise levels direct differentiation of the data $Q_{m}^{\prime}(t)$ provides an acceptable estimate of the true derivative. This is not true for the higher noise level illustrated, and in this case the mollification technique described in $\S 3$ is essential. The mollified derivative is utilised in the deconvolution algorithm (4.3). It is to be observed, by comparison of this figure with Figure 1, the noise am] ion introduced by the differentiation operator.

In Figure 3 the concentration signals $c_{0}(t)$ which have been reconstructed from two $Q_{m}$ signals, with respective noise levels of $2.5 \%$ and $5 \%$, are illustrated. It is again observed, for low noise levels, the direct reconstruction (without mollification) provides an acceptable 
estimate. However, for the higher noise level this is no longer true, and the mollification algorithm is essential. In these results the best values for $\delta$ have been taken, as previously explained; smaller values for $\delta$ produce more oscillations as can be seen from comparison of Figure 3(b) and Figure 4(b), which have $\delta$ values of 150 and 75 , respectively. The larger values of $\delta$ resulting in more smoothing.

It is possible to extend the results in $\S 3$, and find optimum values for $\delta$, given the noise level $\left\|Q_{m}-Q\right\|_{\infty} \leq \epsilon$. However, for the problem this paper addresses, the measurement actually made is $\int_{t_{1}}^{t_{2}} Q(t) d t$ for a sequence of values of $t_{1}$ and $t_{2}$, but with uniform spacing, that is $t_{2}-t_{1}=\Delta t$. It follows that there is little point in choosing $\delta<\Delta t$. The problem where $Q(t)$ is averaged over a sequence of uniformly spaced time periods, say $\Delta t$, will be described in a later paper.

In Figures 3 and Figure 4(b), for the mollified reconstruction presented, it is seen that the reconstructed $c_{0}$ is positive; this is an obvious physical requirement, and this requirement has been added to the algorithm. Observe Figure $4(\mathrm{a})$ which shows the same result as Figure 3(b), but without this constraint; the concentration becomes negative. It is difficult to add such constraints as positivity to the mollification algorithm without resulting in a nonlinear problem.

In order to include positivity in our algorithm therefore, we employ a simple artifice. From the solution of (4.3) we find $l_{b}=\min _{i} \alpha_{i}$, and use as a solution to the deconvolution problem, the modified $\overline{\alpha_{i}}$ values

$$
\overline{\alpha_{i}}=\left\{\begin{array}{ll}
\alpha_{i}, & l_{b}>0, \\
\alpha_{i}-l_{b}, & l_{b}<0,
\end{array} \quad i \in\{0, N\} .\right.
$$

These $\left\{\overline{\alpha_{i}}\right\}_{i=0}^{N}$ are used in the mollified results shown in Figure 3 and Figure 4(b). In Figure 3 and Figure $4(\mathrm{~b})$ no negative concentrations are shown. However, for the un-mollified noisy data, negative values are obtained as shown in Figure 4(a), but they have been suppressed in the other figures.

\section{CONCLUSIONS}

It has been shown that mollification leads to acceptable solutions in the reconstruction of a concentration deconvolution problem. The method proposed is sequential, and computationally efficient, involving only the solution of a triangular system of algebraic equations. The deconvolution procedure advocated should lead to improved interpretation of experimental data in perifusion experiments.

The Newtonian fluid model used in this paper is appropriate for the in vitro experiment described in this paper. However, for in vivo experiments [15] blood is the transporting medium, and for the tube diameters utilised in these experiments blood does not behave in a Newtonian manner. More appropriate fluid velocity profiles for this case will be discussed in another paper.

As discussed in $\S 1$, the Taylor theory would enable an apparent diffusion coefficient to be used to model both the shear and cross-diffusion dispersion effects as a one-dimensional advection-diffusion prc ${ }^{\cdots} \quad$ For the appropriate inverse problem, invariant imbedding techniques similar to those used in [16] could then be used to solve this side-ways heat problem. However, it should be pointed out that the experimental setup, for some of the perifusion apparatus used, seems to be on the borderline of applicability of pure shear dispersion theory, 
as presented in this paper, and the Taylor theory. These ideas will be examined in a later paper.

\section{ACKNOWLEDGEMENTS}

This work was supported by a grant from the Marsden Fund of the Royal Society of New Zealand, and P. R. Shorten acknowledges the receipt of a University of Canterbury postgraduate scholarship. We thank Dr A. J. Sneyd for convincing us that biomathematics is such a rich area of research, and we also thank Drs A. P. LeBeau and A. B. Robson, and Prof. A. E. McKinnon for many helpful discussions on problems involving corticotroph cell modelling.

\section{REFERENCES}

[1] G. I. Taylor. Dispersion of soluble matter in solvent flowing slowly through a tube. Proc. Roy. Soc. London Ser. A, 219:186-203, 1953.

[2] Simon D. Watt and Anthony J. Roberts. The accurate dynamic modelling of contaminant dispersion in channels. SIAM J. Appl. Math., 55(4):1016-1038, August 1995.

[3] G. I. Taylor. Conditions under which dispersion of a solute in a stream of solvent can be used to measure molecular diffusion. Proc. Roy. Soc. London Ser. A, 225:473-477, 1954.

[4] J. E. A. McIntosh and R. P. McIntosh. Influence of the characteristics of pulses of gonadotrophin releasing hormone on the dynamics of luteinizing hormone release from perifused sheep pituitary cells. $J$. Endocr., 98:411-421, 1983.

[5] J. E. A. McIntosh, R. P. McIntosh, and R. J. Kean. Microcomputer-controlled device for delivering hormone stimulation to cell suspensions in perifusion: release of luteinising hormone from sheep pituitary cells. Medical \& Biological Engineering \& Computing, pages 259-262, 1984.

[6] W. R. Smith, G. C. Wake, J. E. A. McIntosh, R. P. McIntosh, M. Pettigrew, and R. Kao. Mathematical analysis of perifusion data: models predicting elution concentration. Am. J. Physiol., 261(1):R247-R256, 1991.

[7] W. R. Smith and G. C. Wake. Mathematical analysis: An inverse problem arising in convective-diffusive flow. IMA J. Appl. Math., 45:225-231, 1990.

[8] David J. N. Wall and Jonas Lundstedt. Inverse source problems involving the one-way wave equation: Source function reconstruction. Wave Motion, 27:55-77, 1998.

[9] T. John Connolly and David J. N. Wall. On some inverse problems for a nonlinear transport equation. Inverse Problems, 13(2):283-295, 1997.

[10] L. D. Landau and E. M. Lifshitz. Fluid Mechanics, volume 6 of Course of Theoretical Physics. Pergamon Press, Oxford, 1959.

[11] P. Linz. Analytical and Numerical Methods for Volterra Equations. SIAM, Philadelphia, 1985.

[12] Diego A Murio. The Mollification Method and the Numerical Solution of Ill-Posed Problems. John Wiley \& Sons, Inc, New York, N.Y., 1993.

[13] M. J. Evans, A. G. Marshall, N. E. Kitson, K. Summers, J. H. Liversey, and R. A. Donald. Factors affecting ACTH release from perifused equine anterior pituitary cells. J. Endocr., 137:391-401, 1993.

[14] M. J. Evans, N. E. Kitson, J. H. Liversey, and R. A. Donald. The effect of cortisol on the secretion of ACTH by anterior pituitary cells of the horse in culture. J. Endocr., 137:403-412, 1993.

[15] S. L. Alexander, C. H. G. Irvine, J. H. Liversey, and R. A. Donald. The effect of isolation stress on the concentrations of arginine vasopressin, $\alpha$-melanocyte-stimulating hormone and ACTH in the pituitary venous effuent of the _......... horse. J. Endocr., 116:325-334, 1988 .

[16] D. J. N. Wall. Signal restoration after transmission through a diffusive medium. In B. J. Noye, M. D. Teubner, and A. W. Gill, editors, Computational Techniques and Applications: CTAC97, pages 711-718, Singapore, 1998. World Scientific. 